\title{
NICHE HYPERGRAPHS OF PRODUCTS OF DIGRAPHS
}

\author{
Martin SonNTAG \\ Faculty of Mathematics and Computer Science \\ TU Bergakademie Freiberg \\ Prüferstraße 1, D-09596 Freiberg, Germany \\ e-mail: sonntag@tu-freiberg.de \\ AND \\ Hanns-Martin TeicherT \\ Institute of Mathematics \\ University of Lübeck \\ Ratzeburger Allee 160, D-23562 Lübeck, Germany \\ e-mail: teichert@math.uni-luebeck.de
}

\begin{abstract}
If $D=(V, A)$ is a digraph, its niche hypergraph $N \mathcal{H}(D)=(V, \mathcal{E})$ has the edge set $\mathcal{E}=\left\{e \subseteq V|| e \mid \geq 2 \wedge \exists v \in V: e=N_{D}^{-}(v) \vee e=N_{D}^{+}(v)\right\}$. Niche hypergraphs generalize the well-known niche graphs and are closely related to competition hypergraphs as well as common enemy hypergraphs. For several products $D_{1} \circ D_{2}$ of digraphs $D_{1}$ and $D_{2}$, we investigate the relations between the niche hypergraphs of the factors $D_{1}, D_{2}$ and the niche hypergraph of their product $D_{1} \circ D_{2}$.
\end{abstract}

Keywords: niche hypergraph, product of digraphs, competition hypergraph.

2010 Mathematics Subject Classification: 05C65, 05C76, 05C20.

\section{REFERENCES}

[1] J. Bang-Jensen and G. Gutin, Digraphs: Theory, Algorithms and Applications (Springer, London, 2001).

[2] C. Cable, K.F. Jones, J.R. Lundgren and S. Seager, Niche graphs, Discrete Appl. Math. 23 (1989) 231-241. doi:10.1016/0166-218X(89)90015-2 
[3] J.E. Cohen, Interval graphs and food webs: a finding and a problem, RAND Corp. Document 17696-PR (Santa Monica, CA, 1968).

[4] M. Cozzens, Food webs, competition graphs and habitat formation, Math. Model. Nat. Phenom. 6 (2011) 22-38. doi: $10.1051 / \mathrm{mmnp} / 20116602$

[5] C. Garske, M. Sonntag and H.-M. Teichert, Niche hypergraphs, Discuss. Math. Graph Theory 36 (2016) 819-832. doi:10.7151/dmgt.1893

[6] S.-R. Kim, The competition number and its variants, in: Quo Vadis, Graph Theory?, Ed(s) J. Gimbel, J.W. Kennedy and L.V. Quintas (Ann. Discrete Math. 55, North Holland, Amsterdam, 1993). doi:10.1016/S0167-5060(08)70396-0

[7] S.-R. Kim, B. Park and Y. Sano, The competition number of the complement of a cycle, Discrete Appl. Math. 161 (2013) 1755-1760. doi:10.1016/j.dam.2011.10.034

[8] S.-R. Kim, B. Park and Y. Sano, A generalization of Opsut's result on the competition numbers of line graphs, Discrete Appl. Math. 181 (2015) 152-159. doi:10.1016/j.dam.2014.10.014

[9] J. Kuhl, Transversals and competition numbers of complete multipartite graphs, Discrete Appl. Math. 161 (2013) 435-440. doi:10.1016/j.dam.2012.09.012

[10] B.-J. Li and G.J. Chang, Competition numbers of complete r-partite graphs, Discrete Appl. Math. 160 (2012) 2271-2276. doi:10.1016/j.dam.2012.05.005

[11] J.R. Lundgren, Food webs, competition graphs, competition-common enemy graphs and niche graphs, in: Applications of Combinatorics and Graph Theory to the Biological and Social Sciences, Ed(s) F. Roberts (IMA 17, Springer, New York 1989) 221-243.

[12] B.D. McKey, P. Schweitzer and P. Schweitzer, Competition numbers, quasi line graphs and holes, SIAM J. Discrete Math. 28 (2014) 77-91. doi:10.1137/110856277

[13] B. Park and Y. Sano, On the hypercompetition numbers of hypergraphs, Ars Combin. 100 (2011) 151-159.

[14] B. Park and Y. Sano, The competition numbers of ternary Hamming graphs, Appl. Math. Lett. 24 (2011) 1608-1613. doi:10.1016/j.aml.2011.04.012

[15] B. Park and Y. Sano, The competition number of a generalized line graph is at most two, Discrete Math. Theor. Comput. Sci. 14 (2012) 1-10.

[16] J. Park and Y. Sano, The double competition hypergraph of a digraph, Discrete Appl. Math. 195 (2015) 110-113. doi:10.1016/j.dam.2014.04.001 
[17] Y. Sano, On hypercompetition numbers of hypergraphs with maximum degree of at most two, Discuss. Math. Graph Theory 35 (2015) 595-598. doi:10.7151/dmgt.1826

[18] D.D. Scott, The competition-common enemy graph of a digraph, Discrete Appl. Math. 17 (1987) 269-280.

doi:10.1016/0166-218X(87)90030-8

[19] M. Sonntag and H.-M. Teichert, Competition hypergraphs, Discrete Appl. Math. 143 (2004) 324-329.

doi:10.1016/j.dam.2004.02.010

[20] M. Sonntag and H.-M. Teichert, Competition hypergraphs of products of digraphs, Graphs Combin. 25 (2009) 611-624.

doi:10.1007/s00373-005-0868-9

[21] M. Sonntag and H.-M. Teichert, Products of digraphs and their competition graphs, Discuss. Math. Graph Theory 36 (2016) 43-58.

doi:10.7151/dmgt.1851

Received 14 March 2017

Revised 13 March 2018

Accepted 13 March 2018 